\title{
Cómo perciben las compe- tencias informacionales los estudiantes universitarios españoles: un estudio de caso
}

\author{
María Pinto* \\ David Guerrero-Quesada**
}

Artículo recibido:

27 de abril de 2014

Artículo aceptado:

15 de abril de 2016

\section{Resumen}

El estudio se centra en un diagnóstico de las competencias informacionales basado en la percepción por parte de los estudiantes de la Universidad de Granada de las cinco ramas del conocimiento (Artes y Humanidades, Ciencias, Ciencias de la Salud, Ciencias Sociales y Jurídicas, e Ingeniería y Arquitectura). Se emplea una metodología cuantitativa apoyada en la encuesta validada IL-HUMASS. Los resultados destacan la importancia que para estos estudiantes tienen las competencias informacionales así como las lagunas de formación que perciben tener en la adquisición de ciertas habilidades, especialmente de aquellas relacionadas con el tratamiento y la comunicación de información; las notables

* Universidad de Granada, España.

mpinto@ugr.es

** Universidad de Granada, España. 
diferencias en actitud y comportamiento informacional entre los estudiantes de los distintos grados, tanto a nivel de autoeficacia percibida como en cuanto a los hábitos de aprendizaje de dichas competencias.

Palabras clave: Alfabetización Informacional; Autoevaluación; Competencias informacionales; Habilidades Informacionales; Cultura disciplinar; Comportamiento informacional.

\section{How Spanish University Students Perceive Informa- tion Competencies: A Case Study \\ María Pinto and David Guerrero-Quesada}

\section{Abstract}

The study focuses on an assessment of information skills based on the perception of students of the University of Granada enrolled in Arts and Humanities, Science, Health Sciences, Social Sciences and Law, and Engineering and Architecture. A quantitative methodology based on the validated survey IL-HUMASS is employed. The results highlight the importance that these students afford to the information competencies and the lack of training they perceive regarding the acquisition of certain skills, especially those related to the processing and communication of information. Results also show significant differences in information behavior and attitude between students enrolled $\mathrm{n}$ different programs, both in terms of self-efficacy and learning habits. It concludes with a SWOT analysis describing the skills valued least and most by students, and a with number of suggestions for improving weaknesses.

Keywords: Information Literacy; Self-Assessment; Information Skills; Information Behavior; Information Competences; Discipline Culture.

\section{INTRODUCCIÓN}

- 1 interés de la Unión Europea y de la Unesco por las investigaciones cen- 
la vida y su impacto en el aprendizaje es creciente. Hoy día, con los cambios que ha supuesto el modelo de convergencia europea, la figura del profesor se ha desplazado hacia el rol de tutor y el centro de atención está en el estudiante, en su capacidad para aprender a aprender y aprender haciendo. Otros factores, también responsables, son la aceleración de la internacionalización de la enseñanza, la rapidez de los progresos científicos y tecnológicos, en particular de las tecnologías de la información y la comunicación, y la creciente complejidad de las distintas profesiones. Existe un consenso generalizado al respecto de que los ciudadanos deben estar preparados para afrontar el aprendizaje a lo largo de la vida con el fin de hacer frente a los desafíos y evitar los peligros de la exclusión social. De ahí que la mayoría de los responsables educativos han redefinido los objetivos didácticos en términos de competencias, entendidas como la capacidad de aplicar los conocimientos

y las destrezas en diversos contextos. Entre ellas, ocupan un lugar medular las competencias informacionales (CI) e informáticas. Por tanto, las universidades españolas tienen un doble reto en esta sociedad de la información: por un lado, avanzar hacia e-Universidades, contando con una adecuada infraestructura e infoestructura informacional, en un contexto equitativo y sostenible; por otro, constituirse en una organización cuya misión y visión derive hacia el beneficio de la sociedad, que transforme sus roles y procesos para que, acorde a los requerimientos de esta sociedad de la información y del conocimiento, pueda afrontar de manera transversal la nueva formación por competencias, con especial protagonismo de las CI, de los estudiantes.

\section{OBjetivos}

El objetivo principal es ofrecer un autodiagnóstico a modo de radiografía del nivel de CI percibidas por una amplia muestra de estudiantes universitarios que comienzan sus estudios en diversas especialidades. Este análisis, a modo de estudio piloto, se ha centrado en titulaciones de la rama de Ciencias Sociales y Jurídicas que se imparten en la Universidad de Granada, y se basa en las opiniones de los estudiantes sobre sí mismos mediante la aplicación de un cuestionario de autoevaluación, de manera que sirva de punto de partida para estudios posteriores de evaluación basados en modelos objetivos. Asimismo, se detectan cuáles son las competencias mejor y peor valoradas por los estudiantes según las ramas de conocimiento. Por último, se analizará si existen diferencias notables de actitud y comportamiento informacional entre los estudiantes universitarios de las titulaciones participantes en el estudio, con el propósito de dar a conocer esos datos a la comunidad académica 
para una adecuada planificación y desarrollo en los curriculos del aprendizaje de las CI.

\section{REVISIÓN DE LA LITERATURA}

La literatura sobre evaluación de CI en la educación superior es muy amplia y, en general, se solapa con la evaluación de los resultados de aprendizaje en el contexto de la ALFIN. La mayoría de los estudios ponen de manifiesto la necesidad de incorporar la gestión de la información, y por tanto la formación en CI, como una actividad transversal para todo estudiante universitario, ${ }^{1}$ y de hacerlo mediante un enfoque basado en la adquisición de competencias, contextualizado en su entorno concreto de uso y retroalimentado con la perspectiva de los usuarios en contexto (Bruce, 1997).

Dado el carácter complejo de la competencia informacional, que implica la capacidad de usar integradamente diferentes habilidades, valores y conocimientos en contextos concretos para resolver problemas o conseguir fines propuestos, la evaluación óptima sería a través de métodos cualitativos, como la entrevista (Monroe-Gulick y Petr, 2012), el análisis de portafolios y de los resultados de las tareas informacionales y de investigación de acuerdo con rúbricas. De estos métodos hay ejemplos significativos (Leichner et al., 2014), pero tienen dificultades de diseño y de aplicación.

Frente a la evaluación cualitativa, los sistemas de evaluación más usados se basan en test o pruebas estandarizadas; ${ }^{2}$ tienen ventajas como la facilidad de aplicación para obtener puntuaciones objetivas, pero pueden quedarse en una evaluación formal, no significativa. Además, implican costo, falta de flexibilidad, y a veces requieren un tiempo excesivo en el proceso de aplicación de los cuestionarios (Goebel et al., 2013).

En la enseñanza universitaria a nivel internacional se han desarrollado numerosos proyectos de evaluación de la alfabetización informacional. ${ }^{3}$ Otros han realizado un cuidadoso análisis crítico de la lógica subyacente de los procesos de evaluación de la ALFIN en la educación superior (Oakleaf y Kaske, 2009). Mención especial merecen también algunos instrumentos homologados para la evaluación de las CI que emplean distintas universidades: Indiana University (Kuh y Gonyea, 2003); ISST, James Madison University

1 Ejemplos de estos estudios son Leu y Kinzer (2005), Mark y Boruff-Jones (2003), Kuiper, Volman y Terwel (2005), Alvermann, Swafford y Montero (2004), Katz (2007), Baildon y Baildon (2008) y Rumsey (2008).

2 Tal y como se describe en Pinto et al. (2013a) y en Blevens (2012).

3 Valgan como ejemplo los llevados a cabo por Sonntag (2008), el de Larkin y Pines (2005) y el de Markless y Streatfield (2007). 
(Cameron y Feind, 2001); Information Literacy Test (ILT) (Cameron, Wise y Lottridge, 2007) que surge en 2002 a partir del ISST con el objetivo de medir las cinco CI de las normas ACRL; Standardized Assessment of Information Literacy Skills (SAILS, http://www.projectsails.org), originalmente desarrollado por la Universidad Estatal de Kent y aprobado en 2003 por Association of Research Libraries (ARL), se basa en las normas ALFIN de ACRL; Education Testing Service (ETS) (2008), elaborado desde la Universidad del Estado de California (CSU), se ha pilotado durante varios años en diversas facultades y universidades; Information Skills Survey (ISS) (Bundy, 2004) es una encuesta que, a tono con las normas australianas de ALFIN y a modo de autoinforme, pide al encuestado que describa qué hace con la información, aportando cierta evidencia de lo que realiza en la práctica; IL-HUMASS ${ }^{4}$ es una encuesta dirigida a autoevaluar y conocer las percepciones y expectativas sobre las CI entre los estudiantes universitarios; INFOLITRANS, ${ }^{5}$ centrado en la evaluación y el aprendizaje de las CI de los estudiantes de Traducción e Interpretación. Otro estudio interesante se centra en el grado de aprendizaje conseguido en competencias formativas básicas como el razonamiento crítico, el razonamiento complejo o la escritura (Arum y Roksa, 2011).

\section{Autoevaluación de las competencias informacionales}

A pesar del argumento de que los avances tecnológicos han facilitado el acceso a la información, hay una continua preocupación ante el hecho de que los estudiantes universitarios no están suficientemente infoalfabetizados: manejan cantidades abrumadoras de información, pero no saben qué hacer con ella, cómo evaluarla, cómo usarla y cómo aprovecharla de forma estratégica y ética. Desconocen cómo abordar de manera sistemática la realización de trabajos académicos y cómo exponer adecuadamente en público.

Parece recomendable que, previo a cualquier tarea de evaluación de competencias, se disponga de un autodiagnóstico que refleje las percepciones de los estudiantes sobre el tema, porque con ello se dirigirán mejor hacia sus carencias y, a través del proceso de análisis de su autovaloración, percibirán la amplitud de la competencia. En esta línea giran las aportaciones de algunos estudios, que describen la visión de los estudiantes sobre la adquisición de CI, la autopercepción de su propio conocimiento base y sus habilidades, para posteriormente comparar esta visión con las puntuaciones que obtienen en un test normalizado. Gross y Latham (2009) compararon las valoraciones

4 Desarrollado en los estudios de Pinto (2010 y 2011).

5 Desarrollado en los estudios de Pinto y Sales(2007 y 2008). 
obtenidas en entrevistas con el análisis de los resultados del cuestionario Information Literacy Test (ILT) para identificar qué diferencias había entre las percepciones de los estudiantes de sus competencias y sus capacidades objetivas. Pinto (2010) plantea el modelo IL-HUMASS para analizar por primera vez la valoración, el autoconcepto y la forma declarada de aprender las competencias informacionales de los estudiantes universitarios españoles de grado. Demiralay y Karadeniz (2010) aplicaron un test denominado Information Literacy Self-efficacy Scale (ILSES) para examinar la percepción de la propia competencia. Comas et al. (2011) estudiaron también la autovaloración de sus competencias informacionales de 1025 estudiantes universitarios de la Universidad de las Islas Baleares, concluyendo que éstos consideraban positivamente sus habilidades en los procesos de información en Internet, a diferencia de sus competencias en el uso de los recursos bibliotecarios. Los resultados revelan una visión general de la alfabetización informacional centrada en el producto más que en el proceso, la percepción de conseguir las CI por cuenta propia, la primacía de la persona sobre las fuentes de información, y el énfasis en el interés personal como la clave en la búsqueda de información.

\section{Metodología}

Pese a que numerosos estudios ponen de manifiesto la importancia de desarrollar una evaluación diagnóstica de las CI, se observa que no existen instrumentos para este fin en el contexto de la enseñanza superior en España. Por eso, para recabar información sobre este tema fue necesario aproximarnos a través de una herramienta de evaluación diseñada ex profeso, IL-HUMASS, ${ }^{6}$ que está siendo completada con el diseño de otros instrumentos de evaluación (Pinto, 2012). El diseño del cuestionario IL-HUMASS ${ }^{7}$ se apoya en un amplio corpus de literatura dentro del campo de la alfabetización informacional internacional, tanto de carácter general como normativo, así como otros de carácter más específico, orientados desde la perspectiva del usuario y desde un enfoque evaluativo. Asimismo, se han revisado otros test dirigidos a conocer las CI de los estudiantes universitarios: SAILS (Kent State University), ISS (Catts, 2005), iSkills (Educational Testing Service, 2008).

A través de 26 variables (subcompetencias), el cuestionario recoge información sobre estas cuatro CI transversales: búsqueda de información, evaluación, procesamiento y comunicación-difusión. Cada una de las variables 
fue evaluada a partir de tres dimensiones: importancia, a través de una escala tipo Likert de nueve puntos; autoeficacia o nivel de logro, usando la misma escala, y bábito preferente de aprendizaje, en el que se presentaban cuatro escenarios: clases, cursos, biblioteca y autodidacta/individual. El cuestionario tiene también una pregunta abierta de carácter cualitativo, que en este estudio no ha sido tratada.

La importancia concedida por el estudiante a cada una de las competencias analizadas supone una acción positiva por parte del mismo, y en todo caso subjetiva, personal e intransferible. Por el contrario, la autoeficacia percibida por el estudiante en relación con cada una de las competencias supone más bien una reacción ante un tema mucho más concreto, como es la puesta en práctica de determinadas acciones encaminadas a conseguir ciertos objetivos informacionales. La dimensión cualitativa denominada hábito preferente de aprendizaje trata de determinar la manera más habitual de aprender o adquirir cada una de las competencias. La elección de ese modo prioritario de aprender no es excluyente, y no implica que el estudiante no utilice otros caminos para el aprendizaje de la competencia en cuestión.

\section{Recolección de datos}

Los encuestados fueron estudiantes de la Universidad de Granada matriculados en 17 títulos oficiales, asegurando que fueran representativos de las cinco ramas del conocimiento. Estos títulos fueron escogidos por la facilidad de acceso a los encuestados por parte de los miembros del equipo de investigación. Se ha priorizado la rama de Ciencias Sociales y Jurídicas, ya que es el área con más estudiantes y la más cercana a nuestra práctica habitual.

El proceso de muestreo elegido fue el probabilístico estratificado para cada uno de los títulos seleccionados previamente. Se asignó un error muestral de $\pm 5 \%$, con un nivel de confianza de $95 \%$. El cálculo del tamaño de las muestras (muestra aleatoria simple) fue realizado con el programa estadístico Stats ${ }^{\mathrm{TM}}$ 2.0, obteniendo un resultado preliminar de $n=1036$ (unidades de muestreo). A partir del criterio sugerido por Malhotra (2004) para el cálculo anticipado de tasa de cumplimiento, partimos de una estimación de al menos un $70 \%$ de cuestionarios respondidos satisfactoriamente, por lo que de manera preventiva incrementamos el tamaño de la muestra hasta lograr un total de 1530 cuestionarios aplicados, de los que 110 fueron descartados resultando una muestra de $n=1420$ (Figura 1).

El cálculo para muestras probabilísticas estratificadas por título universitario se llevó a cabo a partir de la fórmula de Kish. 


\begin{tabular}{|c|c|c|c|}
\hline & $\begin{array}{l}\text { UNIVERTSIUAAUUEZC } \\
\text { Curso academico } 20092010\end{array}$ & Total & Muestra \\
\hline & $\begin{array}{l}\text { Universo de estudiantes en titulos selecocona- } \\
\text { dos }\end{array}$ & 20.722 & 1420 \\
\hline 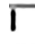 & \multicolumn{3}{|c|}{ ARTESYHUMANIDAOES } \\
\hline & Total de estudiantes & 7723 & 386 \\
\hline & Estudiantes en títulos seleccionados & 3998 & 424 \\
\hline$\Gamma$ & Estudios Ingleses (Filologia Ingless) & 775 & 114 \\
\hline $\begin{array}{l}2 \\
3 \\
4\end{array}$ & $\begin{array}{l}\text { Filologia Hispánica } \\
\text { Historia } \\
\text { Traducción e interpretación }\end{array}$ & $\begin{array}{c}712 \\
792 \\
1719\end{array}$ & $\begin{array}{c}49 \\
103 \\
158\end{array}$ \\
\hline $\mathbb{\pi}$ & \multicolumn{3}{|c|}{ CIENCIAS } \\
\hline 5 & $\begin{array}{l}\text { Total de estudiantes } \\
\text { Estudiantes en títulos seleccionados } \\
\text { Qologia }\end{array}$ & $\begin{array}{l}5015 \\
2632 \\
1380\end{array}$ & $\begin{array}{l}251 \\
108 \\
46\end{array}$ \\
\hline 6 & Matemáticas & 352 & 27 \\
\hline 7 & Medio Ambiente (Ciencias Ambientales) & 900 & 35 \\
\hline II & \multicolumn{3}{|c|}{ CIENCIAS SOCIALESY JURIDICAS } \\
\hline 8 & $\begin{array}{l}\text { Total de estudiantes } \\
\text { Estudiantes en titulos seleccionados } \\
\text { Derecho }\end{array}$ & $\begin{array}{l}26056 \\
6028 \\
3227\end{array}$ & $\begin{array}{l}1303 \\
537 \\
112\end{array}$ \\
\hline 9 & Documentacion & 306 & 79 \\
\hline 0 & Magisterio & 629 & 75 \\
\hline 1 & Psicologia & 1866 & 271 \\
\hline $\bar{v}$ & \multicolumn{3}{|c|}{ CIENCIAS DELA SALUD } \\
\hline & Total de estudiantes & 6568 & 328 \\
\hline & Estudiantes en titulos seleccionados & 2126 & 109 \\
\hline 2 & Medicina & 1627 & 63 \\
\hline 3 & Odontologia & 499 & 46 \\
\hline v & \multicolumn{3}{|c|}{ INGENIERIA Y ARQUITECTURA } \\
\hline & Total de estudianies & 9132 & 457 \\
\hline & Estudiantes en titulos seleccionados & 5798 & 242 \\
\hline 4 & Ingenieria civil (de Caminos) & 1622 & 70 \\
\hline 5 & Ingerieria de edificacion (Asquitectura Técnica) & 2487 & 86 \\
\hline 6 & Ingenieria informática & 539 & 39 \\
\hline 7 & Ingenieria Quimica & 1150 & 47 \\
\hline
\end{tabular}

Figura 1. Títulos seleccionados por rama del conocimiento

De los 1420 estudiantes encuestados, 914 fueron mujeres y 506 hombres. La recolección de datos abordó todos los cursos, con mayor énfasis en los cursos primero ( $38.6 \%$ de la muestra), tercero ( $30.9 \%$ ) y quinto $(17.1 \%)$.

El cuestionario se aplicó entre los meses de enero y mayo de 2010, y siempre que fue posible se administró en línea en los laboratorios de informática de la universidad, con el consentimiento de los profesores implicados. Los 
cuestionarios se rellenaron en presencia de un miembro del equipo de investigación, quien se encargaba de solventar cualquier duda con relación a la cumplimentación del cuestionario o el uso de la plataforma online. El protocolo de instrucciones fue aplicado por los mismos investigadores en cada uno de los centros para eliminar la posibilidad de sesgos en las respuestas.

\section{Análisis}

Para comprobar la fiabilidad se aplicó el coeficiente alfa de Cronbach, que arrojó un valor medio de correlación entre las variables de 0.948 , lo cual permite asegurar que el cuestionario es fiable. Se confirma también la fiabilidad de la muestra si observamos las correlaciones significativas (fórmula de Pearson) existentes entre las cuatro categorías de competencias que forman parte de este modelo auto-evaluador (Figura 2). Puede verse cómo todas las categorías están correlacionadas de un modo significativo en las dimensiones de importancia y autoeficacia.

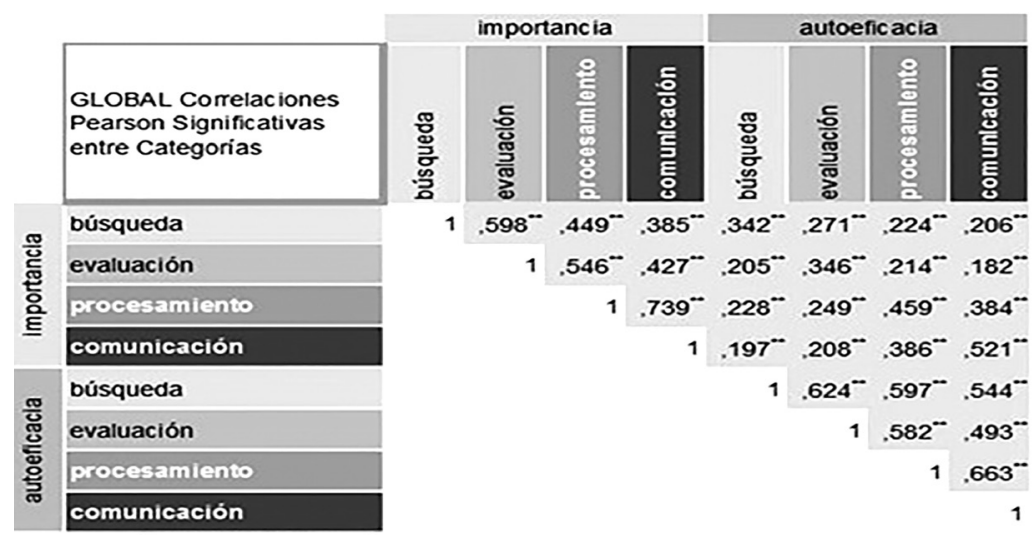

Figura 2. Mapa de correlaciones significativas entre categorías

En resumen, los resultados obtenidos tras el análisis de correlación indican no sólo que se trata de un instrumento con una alta consistencia interna, muy cohesionado y fiable para la recolección de datos, sino también que la muestra seleccionada es homogénea, consistente y fiable. En cuanto a la validez de los datos, tanto los correspondientes a las dimensiones cuantitativas (importancia y autoeficacia) como los pertenecientes a la dimensión cualitativa (hábitos de aprendizaje) se han validado estadísticamente. En cualquier caso, la correlación significativa entre las dimensiones de motivación y autoeficacia ya ha sido demostrada previamente en muestreos similares (Pinto, 2011). 
Acorde con los objetivos planteados en este trabajo, analizaremos desde una perspectiva global los hallazgos más relevantes en relación con la visión-autopercepción que tienen los estudiantes encuestados acerca de las CI.

Los valores medios obtenidos para las dimensiones importancia y autoeficacia de las CI desde una perspectiva global y desde las cuatro categorías competenciales analizadas (búsqueda de información, evaluación, procesamiento y comunicación-difusión) aparecen en la Figura 3. Las puntuaciones globales resultantes ( 7.15 para importancia y 5.89 para autoeficacia) resumen la media de las valoraciones de los estudiantes encuestados en el total de las $26 \mathrm{CI}$ analizadas. Aunque ambas dimensiones ofrecen un nivel general que podemos considerar notable en importancia y aceptable en autoeficacia, es cierto que ambas puntuaciones pueden mejorarse.

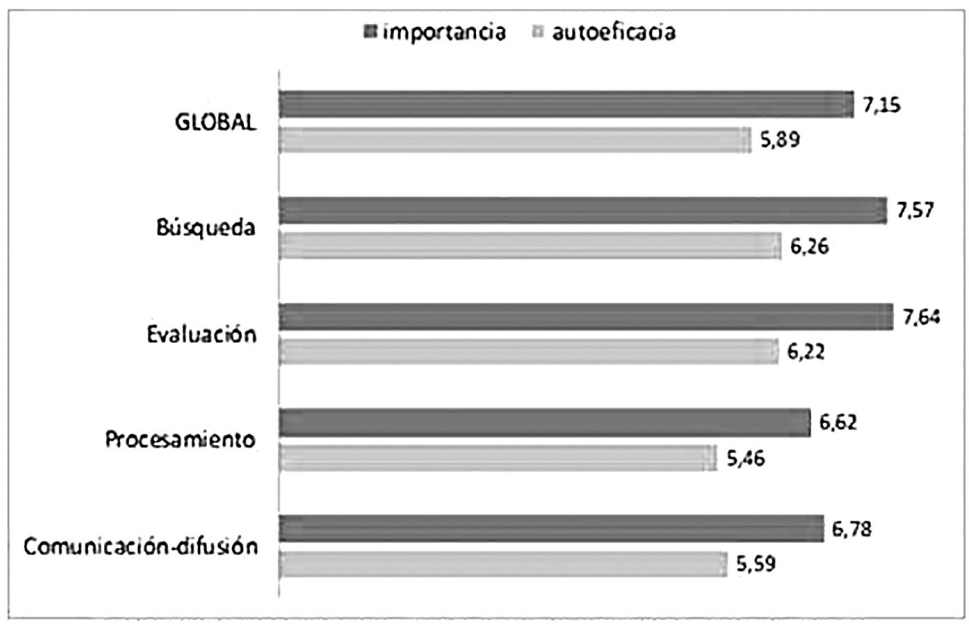

Figura 3. Importancia-autoeficacia global y por categorías infromacionales

En referencia a las cuatro categorías informacionales, surgen dos agrupaciones claramente diferenciadas: por un lado, las categorías de búsqueda y evaluación de la información, cuyas puntuaciones medias en lo que a importancia y autoeficacia se refiere son bastante superiores, por encima de la puntuación global; por otro, las categorías de procesamiento y comunicación-difusión, cuyas apreciaciones son marcadamente inferiores, por debajo de los valores globales. Podemos inferir que los estudiantes encuestados en la 
Universidad de Granada les importa más buscar y evaluar la información, teniendo su autoestima competencial más alta precisamente en estos dos grupos de competencias. Por el contrario, no se encuentran tan estimulados para procesar y comunicar-difundir la información, ni tampoco se consideran eficaces a la hora de poner en práctica las distintas competencias de esta categoría.

Los siguientes diagramas de caja muestran la distribución de los valores en las dimensiones importancia y autoeficacia (Figura 4).

Importancia

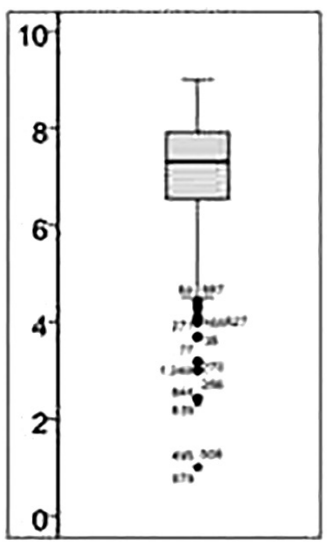

Autoeficacia

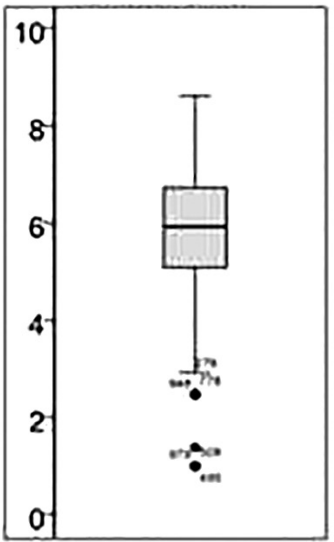

Figura 4. Distribución de valores para las dimensiones importancia y autoeficacia

Se evidencia el grado de concentración-dispersión de los datos obtenidos, así como la presencia-ausencia de valores atípicos. En el diagrama de importancia de las CI los datos se encuentran bastante concentrados, con un cierto grado de asimetría que concentra los datos en sus puntuaciones más altas. Por su parte, el diagrama de autoeficacia percibida está ligeramente más disperso, pero presenta una simetría casi total. En todo caso, aparecen valores atípicos en la parte baja de ambos diagramas. No se observa una evolución de los resultados importancia-autoeficacia por cursos que nos permita inferir un cierto progreso o retroceso en lo que a alfabetización informacional se refiere, pues entre el primer y el quinto curso no se aprecian diferencias significativas, ni tampoco entre cursos intermedios ( Figura 5).

En cuanto a los hábitos de aprendizaje para la adquisición de las competencias, los resultados de este estudio muestran que entre los estudiantes encuestados predomina el autoaprendizaje, seguido por el aprendizaje en clase, 
la biblioteca y los cursos específicos, en este orden. Creemos que la creciente importancia del aprendizaje independiente en los últimos años está ligada de manera estrecha, aunque no excluyente, a la implementación de las tecnologías de la información y la comunicación, y a los nuevos requerimientos del Espacio Europeo de Educación Superior. Si nos centramos en las cuatro categorías de las competencias, esta dimensión muestra algunas diferencias en los patrones de conducta de los estudiantes (Figura 6).

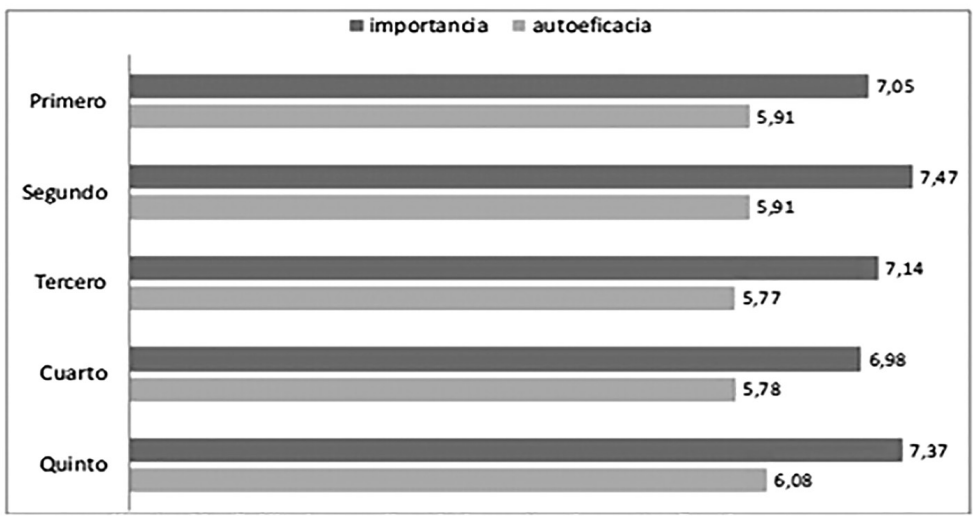

Figura 5. Niveles medio de importancia y autoeficacia por curso
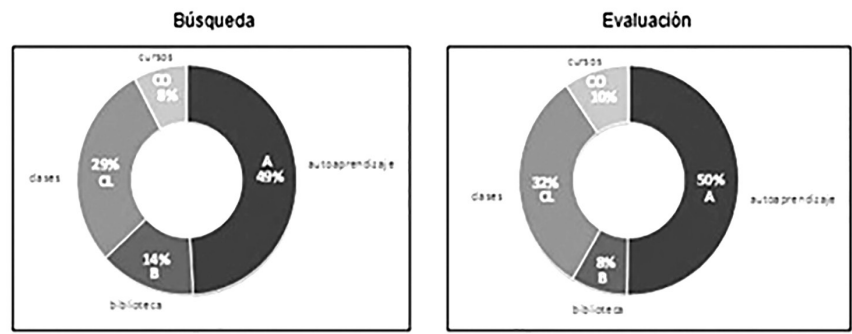

Procesamiento

Comunicación-Difusión
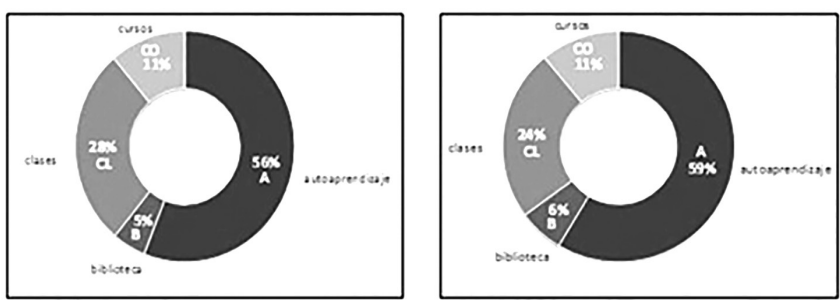

Figura 6. Comparación hábito preferente de aprendizaje por categorías 
El autoaprendizaje se incrementa en las categorías Procesamiento y Comunicación-Difusión, que son precisamente los ámbitos informacionales en los que los pares Importancia-Autoeficacia muestran un mayor grado de debilidad. Se observa que existe en cierto modo un refugio en, o una buida bacia esta modalidad de aprendizaje cuando fallan las CI del estudiante.

Para algunos teóricos del aprendizaje, los estudiantes de ahora practican un mayor uso de Internet debido a la disminución global de las habilidades cognitivas necesarias para el aprendizaje clásico a través de material impreso (Weiler, 2005). La gran mayoría de los estudiantes se dirigen primero a los sitios públicos de Internet en lugar de recurrir a los recursos basados en la biblioteca, a pesar de que muchos de éstos son también accesibles electrónicamente. De este modo eluden el tener que enfrentarse a la comprensión de las complejidades operativas del entorno bibliotecario (Head, 2008).

Otro hallazgo significativo de este estudio de evaluación basado en las autopercepciones de los estudiantes es que podemos identificar las principales fortalezas y debilidades en la adquisición de las CI, con el propósito de poder diseñar acciones formativas que sean incorporadas al proceso de aprendizaje.

Para cada categoría de competencias hemos centrado la atención en los valores extremos obtenidos en las magnitudes Importancia y Autoeficacia expresadas por los estudiantes. En la Figura 7 se muestran las habilidades mejor y peor valoradas. Como puede observarse, el número de competencias consideradas como una debilidad es claramente superior al de las consideradas como fortalezas. La situación más extrema corresponde a la competencia peor valorada por los estudiantes en la encuesta, que es la relativa a la gestión de bases de datos, cuyo valor medio es el más bajo en todas las ramas de conocimiento analizadas.

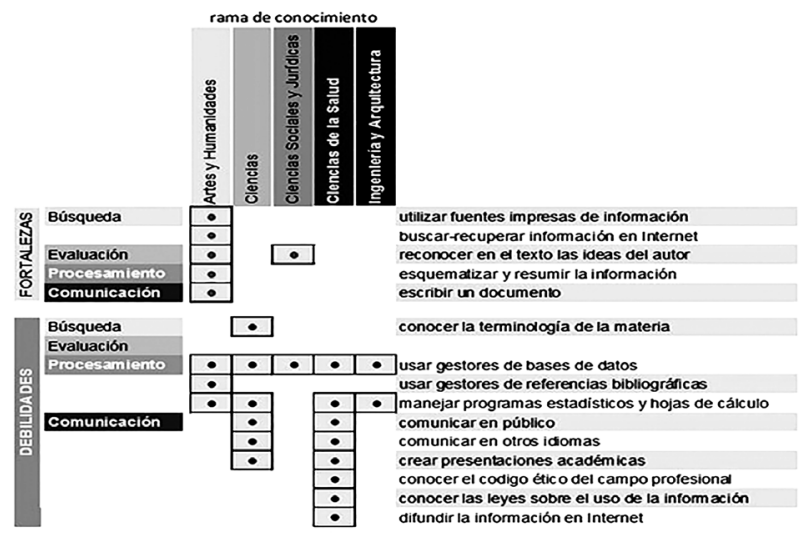

Figura 7. Fortalezas y debilidades por área del conocimiento 
Por otro lado, las fortalezas están distribuidas entre cinco competencias, y se concentran en la rama de Artes y Humanidades. En las otras cuatro ramas no aparecen puntos fuertes, según los criterios adoptados. En resumen, la rama del conocimiento que muestra un mayor nivel de alfabetización, al menos desde el punto de visto subjetivo de los estudiantes encuestados, es la de Artes y Humanidades.

Los puntos fuertes que aparecen al menos en una rama de conocimiento se encuentran repartidos en las cuatro categorías informacionales y corresponden a las siguientes cinco competencias: utilizar fuentes impresas de información, buscar-recuperar información en Internet, reconocer en el texto las ideas del autor, esquematizar y resumir la información y escribir un documento. Su presencia es reducida, no sólo por el limitado número de competencias que han alcanzado el rango de fortalezas informacionales, sino sobre todo porque su implantación en las distintas ramas de conocimiento es muy reducida, afectando prioritariamente a la rama de Artes y Humanidades, y alguna en la de Ciencias Sociales y Jurídicas. Los estudiantes de las otras tres ramas de conocimiento (Ciencias, Ciencias de la Salud e Ingeniería y Arquitectura) no manifiestan fortalezas desde el punto de vista de la alfabetización informacional.

En cuanto a los puntos débiles con presencia en al menos una rama de conocimiento, observamos que la categoría de evaluación de la información no presenta debilidades en la muestra estudiada. Las competencias que muestran debilidades se distribuyen en las otras tres categorías informacionales, y son diez: conocer la terminología de la materia, usar gestores de bases de datos, usar gestores de referencias bibliográficas, manejar programas estadísticos y bojas de cálculo, comunicar en público, comunicar en otros idiomas, crear presentaciones académicas, conocer el código ético del campo profesional, conocer las leyes sobre el uso de la información y difundir la información en Internet. Su presencia se concentra en las ramas de Ciencias y Ciencias de la Salud.

La presencia de datos atípicos demuestra el carácter no reglado de la alfabetización informacional, muy necesitada de su integración en los programas académicos de la universidad. No se observa progreso en el nivel de alfabetización informacional a lo largo de los distintos cursos, y ello es consecuencia de la carencia de una planificación específica en los curriculos.

Estos datos pueden correlacionarse con otras investigaciones que han estudiado las CI entre estudiantes universitarios, especialmente aquellas centradas en la búsqueda y la evaluación de la información. Sirva como referencia el trabajo llevado a cabo con una gran muestra de universitarios norteamericanos y británicos, que concluyó que los estudiantes percibían que tenían una mayor habilidad para buscar información en Internet, 
sobresaliendo ésta como su fuente preferida para encontrar información académica (Borreson y Salaway, 2008).

Por último, el estudio nos ofrece información relevante sobre las posibles diferencias entre los estudiantes de las titulaciones de las cinco ramas de conocimiento analizadas. En la Figura 8 se recogen los valores medios correspondientes a la importancia y la autoeficacia dentro de las cinco ramas del conocimiento.

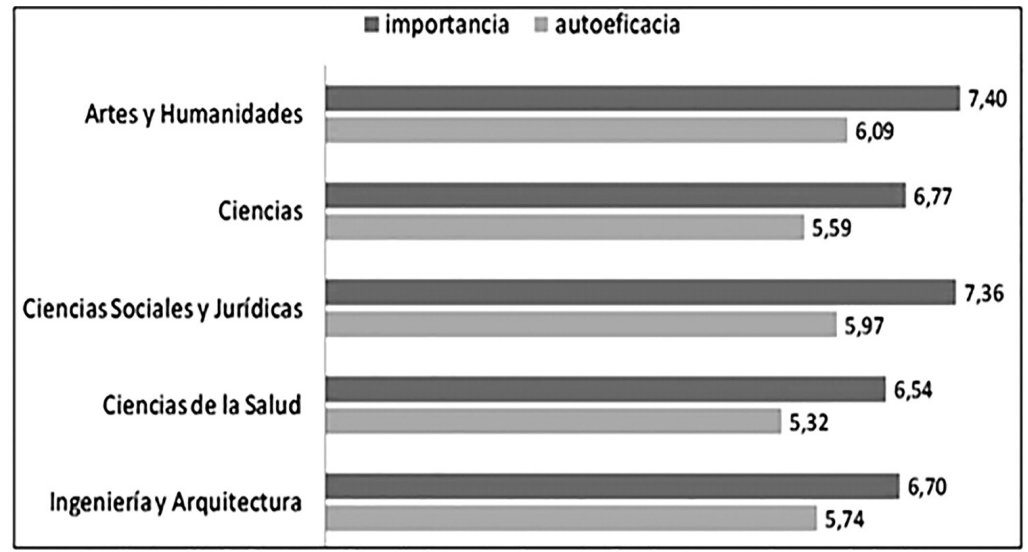

Figura 8. Comparación entre importancia y autoeficacia por rama de conocimiento

En términos del nivel de importancia concedido, dos de las ramas sobresalen sobre la media: Artes y Humanidades, y Ciencias Sociales y Jurídicas. Las otras tres ramas ofrecen unos valores similares entre sí y ligeramente por debajo de la media. Algo similar ocurre con los resultados de autoeficacia. Cabe destacar que la rama con la menor puntuación en estas dos magnitudes es la de Ciencias de la Salud. La distribución de los resultados por rama de conocimiento, reflejada en las Figuras 9 y 10, nos permite confirmar esas diferencias.

De acuerdo con el estudio comparativo sobre hábitos prioritarios de aprendizaje, podemos observar cómo el autoaprendizaje tiene el mayor porcentaje de respuestas, a una gran distancia del resto de opciones, excepto en la rama de Ciencias Sociales y Jurídicas, donde la distancia con la segunda opción, el aprendizaje en el aula, se acorta ostensiblemente. La biblioteca y los cursos específicos ocupan los últimos puestos (Figura 11). 


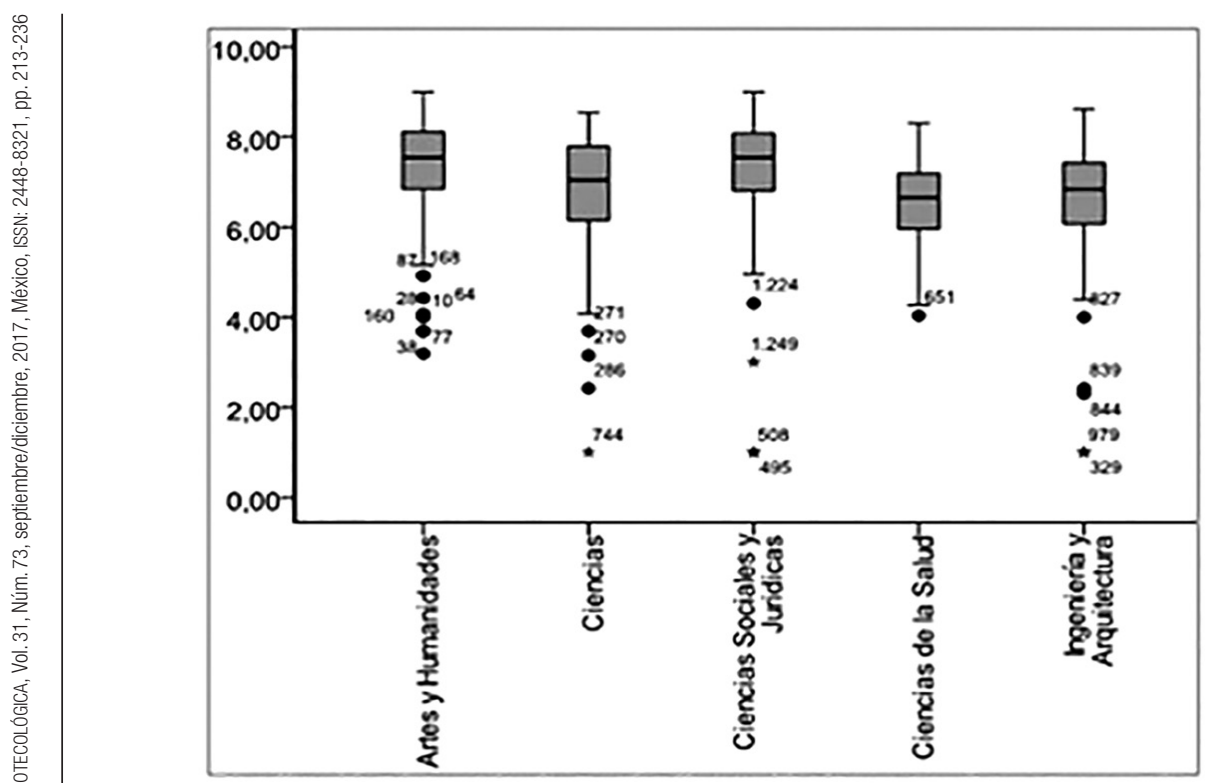

Figura 9. Distribución de resultados en la dimensión importancia por ramas del conocimiento

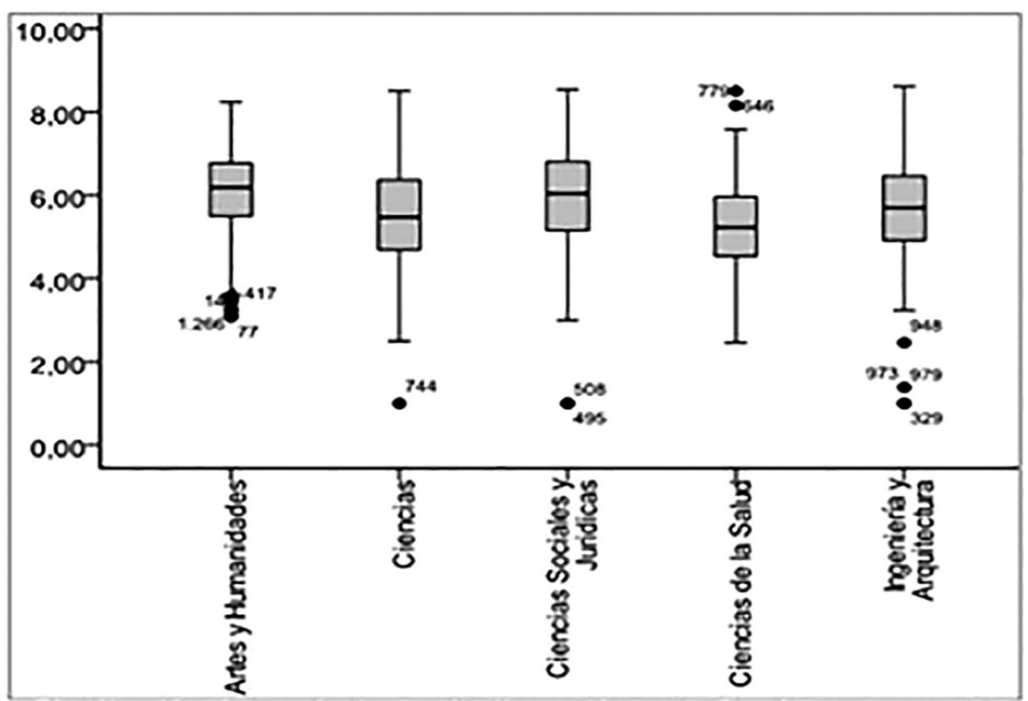

Figura 10. Distribución de resultados en la dimensión autoeficacia por ramas del conocimiento 


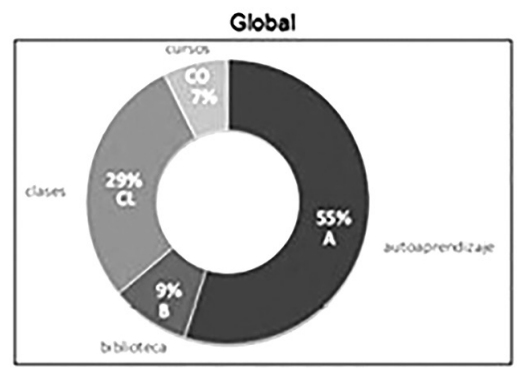

Ciencias do la Salud

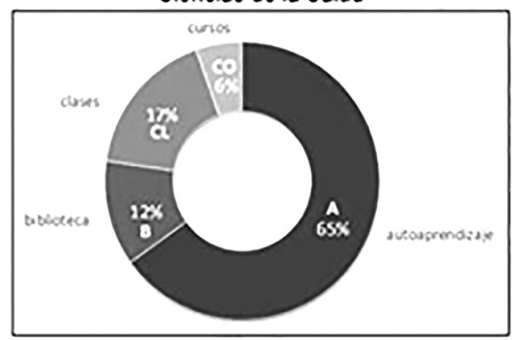

Cioncias

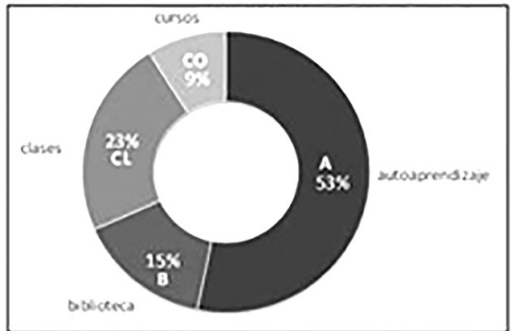

Artes y Humanidades

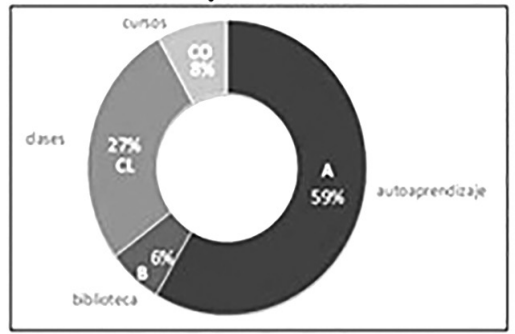

Ingonioria y Arquitoctura

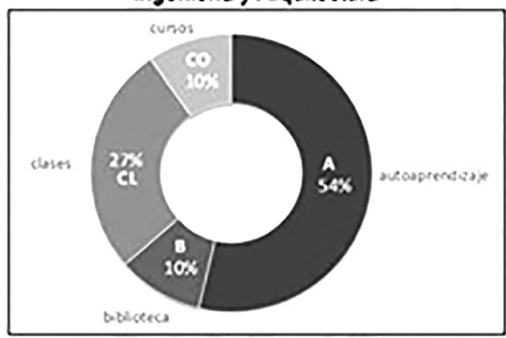

Cioneios Socialos y Juridiess

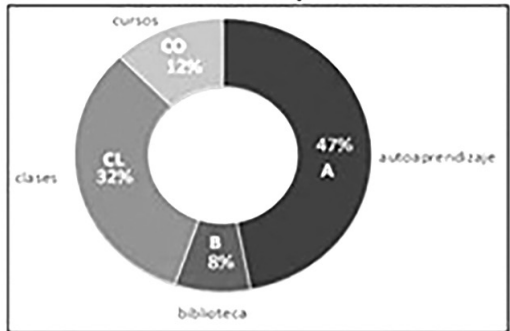

Figura 11. Hábitos prioritarios de aprendizaje: resultados globales y por rama de conocimiento

\section{CONCLUSIONES Y RECOMENDACIONES}

Esta macroinvestigación exploratoria realizada en la Universidad de Granada es pionera a nivel internacional y sin duda constituye un referente para otros trabajos en curso. Su metodología centrada en el uso del instrumento IL-HUMASS validado y confiable puede ser extrapolable a otros entornos universitarios, pues permite diagnosticar cómo perciben los estudiantes universitarios la importancia que tienen para su formación la adquisición de las CI, sus expectativas en cuanto al nivel de logro y dónde dicen que la adquieren. 
Como punto de arranque se centra en la filosofía de la autoevaluación, con el estudiante como la unidad primaria de análisis, aportando una visión interna, y por consiguiente subjetiva, de los procesos de relación del estudiante con el entorno documental en el que, irremisiblemente, se tiene que desenvolver. Esta visión interna y subjetiva constituye el mejor punto de partida para emprender acciones de evaluación objetivas que permitan un diagnóstico integral de la adquisición de las CI, proporcionando pistas claves para la planificación de acciones formativas estratégicas que contribuyan al desarrollo personal, académico, social, etc., del estudiante durante su proceso formativo.

Desarrollado en un entorno bastante distinto, como es el norteamericano, el estudio PIL (Head y Eisenberg, 2010) presenta ciertas similitudes con el nuestro, aunque quizás sea bastante más concreto al centrarse preferentemente en las etapas de búsqueda y evaluación de la información. El protagonismo para las competencias de procesamiento y comunicación-difusión de la misma es escaso. Si comparamos ambos estudios, se observa cómo los estudiantes españoles, al igual que los estadounidenses, muestran resultados positivos tanto en la búsqueda como en la evaluación, pero fallan en el procesamiento y la difusión de la información, debido a una deficiente formación en estos aspectos, los cuales exigen un entrenamiento más profundo y continuado.

La cultura de la alfabetización informacional, y el consiguiente dominio de las CI, afecta tanto al individuo como al entorno académico global en el que desempeña su actividad. En este sentido, todas las personas (gestores, investigadores, profesores, PAS y estudiantes) involucradas en la consecución y en la aplicación de la misión y de los objetivos de la universidad deberían conseguir un nivel adecuado de competencia en gestión, acceso y uso de la información. Una universidad alfabetizada en información planifica sistemáticamente todas sus actividades de formación y de actualización en ALFIN para todos los grupos de interés, por medio de una estrategia institucional claramente establecida que cubra las políticas, los recursos, las infoestructuras y las actividades de desarrollo formativo de sus integrantes (Webber y Johnston, 2006).

La presencia de datos atípicos en los resultados demuestra el carácter no reglado de la alfabetización informacional, muy necesitada de su integración en los programas académicos y curriculos universitarios actuales. Además, el hecho de que no se observe en general un progreso gradual en el nivel de alfabetización informacional a lo largo de los distintos cursos no deja de ser una consecuencia más de la carencia de esa planificación curricular específica.

Para interiorizar adecuadamente el proceso de aprendizaje de las CI, los estudiantes han de aprender a interrelacionar adecuadamente las competencias y habilidades declarativas, centradas en actividades de pensamiento 
de corte cognitivo, tales como analizar, interpretar, resumir, evaluar, diseñar, producir, elaborar, generar, comunicar y transmitir conocimiento, con las competencias y habilidades procedimentales, basadas en actividades y procedimientos rutinarios tales como localizar, recuperar información, instalar programas de ordenador, manejar programas estadísticos y hojas de cálculo, etc. En el actual entorno digital se considera que el dominio de las competencias de comunicación y difusión es vital para optimizar el flujo de información y conocimiento a través de las TIC. En todo caso, ambos tipos de habilidades (declarativas y procedimentales) son esenciales para la alfabetización informacional y necesarias para el aprendizaje a lo largo de la vida.

Otro hallazgo interesante es la constatación de que los estudiantes aseguran que adquieren las CI del autoaprendizaje, en vez de recurrir a formas regladas (cursos) o a la guía que podrían proporcionarles tanto profesores como bibliotecarios.

Habría que potenciar la formación-evaluación de estas CI en las guías docentes de todos los títulos universitarios como parte aplicativa y contextual de los distintos dominios científicos, correlacionándolas con los objetivos-resultados de aprendizaje específicos. Además, se debería llevar a cabo un replanteamiento de la formación ofrecida por las bibliotecas, tradicionalmente centrada en los recursos (Head y Eisenberg, 2010).

Asimismo, resulta significativo que no existan diferencias entre los estudiantes con base en el curso en el que se encuentran, lo cual demuestra que no hay un progreso ni en el dominio de las CI ni en la percepción de la importancia que estos estudiantes dan a dichas competencias. Al no tratarse éste de un estudio longitudinal, no podemos asegurar cuál es la causa de esta ausencia de mejora, aunque se puede aventurar una falta de planificación del proceso de docencia-aprendizaje por parte de los distintos agentes del sistema de educación superior.

Como conclusión, podemos decir que los resultados obtenidos ofrecen notables implicaciones de cara no sólo a la mejora en los niveles de alfabetización informacional de los estudiantes, sino también a la planificación institucional y académica de estas competencias en el marco de los estudios impartidos por las universidades españolas. En el contexto del Espacio Europeo de Educación Superior, la alfabetización en información ha de ser una estrategia significativa para el aprendizaje a lo largo de la vida. El dominio de las CI, como un resultado clave de la educación superior, es una respuesta necesaria a las crecientes demandas de un mercado basado en la economía del conocimiento. Parece que la adquisición y dominio de las CI contribuyen al éxito académico de los titulados, y que los titulados que estén infoalfabetizados tendrán mejor acceso al mercado laboral y a un aprendizaje permanente a lo largo de la vida. 
Agradecimientos

Esta investigación ha sido posible gracias a la financiación previa por parte de la Universidad de Granada, mediante el Programa de Adaptación de las Enseñanzas al Espacio Europeo de Educación Superior (PAE-016), y del Ministerio de Innovación, Ciencia y Deporte, mediante el Subprograma de Proyectos de Investigación Fundamental No Orientada (EDU2011-29290). Queremos agradecer también la colaboración desinteresada y la disponibilidad de los profesores y estudiantes de la UGR en la elaboración de este proyecto.

\section{REFERENCIAS}

Alvermann, D., J. Swafford y M. Montero. 2004. Content area literacy instruction for the elementary grades. Upper Saddle River, NJ: Pearson Education.

Arum, R. y J. Roksa. 2011. Academically Adrift: Limited Learning on College Campuses. Chicago: University of Chicago Press.

Baildon, R. y M. Baildon. 2008. "Guiding Independence: Developing a Research Tool for Support Student Decision Making”. Selecting Online Information Sources. The Reading Teacher 61 (8): 636-647.

Blevens, C. L. 2012. "Catching up with information literacy assessment: Resources for program evaluation”. College E Research Libraries News 73 (4): 202-206.

Borreson, J. y G. Salaway. 2008. The ECAR study of undergraduate students and information technology. Fecha de consulta: 12 de enero de 2010. http://www.educause.edu/ers0808

Bruce, C. 1997. Seven Faces of Information Literacy. Adelaide: Auslib Press.

Bundy, A., ed. 2004. Australian and New Zealand Information Literacy Framework: principles, standards and practice. Adelaida: Australian and New Zealand Institute for Information Literacy.

Cameron, L. y R. Feind. 2001. "An Online Competency Test for Information Literacy: Development, Implementation, and Results”. ACRL Tenth National Conference, March 15-18, 2001, Denver, Colorado.

Cameron, L., S. Wise y S. Lottridge. 2007. "The Development and Validation of the Information Literacy Test”. College and Research Libraries 68 (3): 229-236.

Catts, R. 2005. Information Skills Survey Technical Manual. Canberra: CAUL.

Comas, R., J. Sureda, M. Pastor y M. Morey. 2011. "La búsqueda de información con fines académicos entre el alumnado universitario". Revista Española de Documentación Científica 34 (1): 44-64.

Demiralay, R. y S. Karadeniz. 2010. "The Effect of Use of Information and Communication Technologies on Elementary Student Teachers' Perceived Information Literacy Self-Efficacy". Kuram ve Uygulamda Egitim Bilimleri 10 (2): 841-851.

Educational Testing Service. 2008. iSkills Assessment. Fecha de consulta: 12 de enero de 2011 http://www.ets.org/iskills/about

ENIL (European Network on Information Literacy). S. f. Fecha de consulta: 4 de noviembre de 2010. http://www.ceris.cnr.it/Basili/EnIL/index.html 
Goebel, N., J. Knoch, M. E. Thomson, R. Willson y S. Sharun. 2013. "Making assessment less scary: Academic libraries collaborate on an information literacy assessment model". College \& Research Libraries News 74: 28-31.

González, J. y R. Wagenaar, eds. 2003. Tuning Educational Structures in Europe: Informe Final. Fase uno. Bilbao: Universidad de Deusto y Universidad de Groningen.

Gross, M. y D. Latham. 2009. "Undergraduate perceptions of information literacy: defining, attaining, and self-assessing skills". College \& Research Libraries 70 (4): 336-350.

Head, A. J. 2008. "Information Literacy from the Trenches: How Do Humanities and Social Science Majors Conduct Academic Research?". College \& Research Libraries 69 (5): 427-446.

Head, A. J. y M. B. Eisenberg. 2010. "Project Information Literacy Progress Report: Truth Be Told: How College Students Evaluate and Use Information in the Digital Age". The Information School. University of Washington, Washington.

Ianuzzi, P. 1999. "We Are Teaching, but Are They Learning: Accountability, Productivity and Assessment". The Journal of Academic Librarianship 25 (4): 304-305.

Katz, I. 2007. "TS Research finds college students fall short in demonstrating ICT literacy: National Police Council to create national standards [Educational Testing Service]". College \& Research Libraries News 68 (1): 35-37.

Kuh, G. y R. Gonyea. 2003. "The Role of the Academic Library in Promoting Student Engagement in Learning”. College \& Research Libraries 64 (4): 269-270.

Kuiper, E., M. Volman y J. Terwel. 2005. "The Web as an Information Resource in K12 Education: Strategies for Supporting Students in Searching and Processing Information". Review of Educational Research 75 (3): 285-328.

Larkin, J. E. y H. A. Pines. 2005. "Developing Information Literacy and Research Skills in Introductory Psychology: A Case Study". The Journal of Academic Librarianship 31 (1): 40-45.

Leichner, N., J. Peter, A-K. Mayer y G. Krampen. 2014. “Assessing information literacy programmes using information search tasks". Journal of Information Literacy 8 (1): 3-20.

Leu, D. y C. Kinzer. 2005. "The convergence of literacy instruction with net-worked technologies for information and communication". Reading Research Quarterly 35 (1): $108-127$.

Malhotra, N. 2004. Marketing Research: an Applied Orientation, $4^{a}$ ed., New Jersey: Prentice Hall.

Mark, A. y P. Boruff-Jones. 2003. "Information Literacy and Student Engagement: What the National Survey of Student Engagement Reveals about your Campus". College \& Research Libraries 4 (6): 480-493.

Markless, S. y D. Streatfield. 2007. "Three Decades of Information Literacy: Redefining the parameters", en Change and Challenge: Information Literacy for the 21st Century, S. Andretta, 15-36. Adelaide: Auslib Press.

Monroe-Gulick, A. y J. Petr. 2012. "Incoming graduate students in the social sciences: How much do they really know about library research?”. Portal 12 (3): 315-335.

Oakleaf, M., y N. Kaske. 2009. "Guiding Questions for Assessing Information Literacy in Higher Education". Portal: Libraries and the Academy 9 (2): 273-286. 
Pinto, M. 2010. "Design of the IL-HUMASS Survey on Information Literacy in Higher Education: a self-assessment approach". Journal of Information Science 36 (1): 86-103.

Pinto, M. 2011. "An Approach to the Internal Facet of Information Literacy using the IL-HUMASS Survey”. Journal of Academic Library 37 (2): 145-154.

Pinto, M. 2012. "Information Literacy Perceptions and Behavior among History Students". Aslib Proceedings 64 (3): 304-327.

Pinto, M. y D. Sales. 2007. "A Research Case Study for User-Centered Information Literacy Instruction: Information Behavior of Translation Trainees". Journal of Information Science 33 (5):531-550.

Pinto, M. y D. Sales. 2008. "INFOLITRANS: A Model for the Development of Information Competence for Translator". Journal of Documentation 64 (3): 413-437.

Pinto, M., A. Fernández-Ramos, G. Sánchez y G. Meneses. 2013a. "Information Competence of Doctoral Students in Information Science in Spain and Latin America: A Self-assessment”. Journal of Academic Librarianship 39 (2): 144-154.

Pinto, M., J. Gómez-Hernández, S. Puertas, D. Guerrero, X. Granell, C. Gómez, R. Palomares y A. Cuevas. 2013b. "Designing and Implementing Web-Based Tools to Assess Information Competences of Social Science Students at Spanish Universities". Communications in Computer and Information Science 397: 443-449. CCIS

Rumsey, S. 2008. How to Find Information. Oxford: McGraw Hill.

SAILS. S. f. Project Sails (Standardized Assessment of Information Literacy Skills). Fecha de consulta: 23 de abril de 2011. http://www.projectsails.org

Sonntag, G. 2008. "Los Resultados de los Programas de Alfabetización Informacional: la Evaluación”, en Brecha Digital y Nuevas Alfabetizaciones. El Papel de las Bibliotecas, J. Gómez, A. Calderón y J. Magán, 111-137. Madrid: Biblioteca Universidad Complutense.

Webber, S. y B. Johnston. 2006. "Working Towards the Information Literate University”, en Information Literacy: recognizing the need, G. Walton y A. Pope (eds.), 47-58. Oxford: Chandos.

Webber, S., S. Boon y B. Johnston. 2005. "A Comparison of UK Academics' Conceptions of Information Literacy in Two Disciplines: English and Marketing”. Library E Information Research 29 (93): 4-15.

Weiler, A. 2005. "Information-Seeking Behavior in Generation Y Students: Motivation, Critical Thinking and Learning Theory". Journal of Academic Librarianship 31 (1): 46-53.

Para citar este texto:

Pinto, María y David Guerrero-Quesada. 2017. "Cómo perciben las competencias informacionales los estudiantes universitarios españoles: un estudio de caso". Investigación Bibliotecológica: archivonomía, bibliotecología e información 73 (31): 213-236. http://dx.doi.org/10.22201/iibi.24488321xe.2017.73.57854 


\section{Anexo}

\section{Cuestionario IL-HUMASS utilizado en la realización del estudio}

\section{INFOLIT-HUMASS}

En la actual Sociedad de la Información y la Comunicación, es importante acceder, analizar y utilizar la información adecuadamente. Para que esto se pueda realizar, y tal y como aparece en los postulados del EEES son necesarias una serie de habilidades y competencias relacionadas con la investigación, la evaluación, la gestión, el uso de y la difusión.

Este cuestionario se construye para conocer tu opinión sobre tus propias competencias y habilidades en el manejo y uso de la información. Por favor, señala como puntúa, evalúa cada una de las siguientes competencias seleccionando la afirmación que mejor expresa tu opinión, en una escala creciente de 1 (baja competencia) a 9 (competencia excelente). También te pedimos la evaluación de cada competencia en relación con tres variables (importancia, auto-eficacia y fuente de conocimiento) que se describen a continuación.

\begin{tabular}{|l|l|}
\hline Motivación & $\begin{array}{l}\text { Evalúa la importancia de las siguientes competencias para tu progreso } \\
\text { académico }\end{array}$ \\
\hline Auto-eficacia & Evalúa tu nivel de habilidad en las siguientes competencias \\
\hline Fuente de aprendizaje & $\begin{array}{l}\text { ¿Dónde desarrollas estas competencias? (Clase, biblioteca, cursos, } \\
\text { autodidacta, otros). Selecciona la opción más adecuada }\end{array}$ \\
\hline
\end{tabular}

Ayúdanos a mejorar, en tu viaje formativo la opinión es importante

\begin{tabular}{|l|l|l|c|}
\hline \multicolumn{1}{|c|}{ En referencia a... } & Importancia & Autoeficacia & $\begin{array}{c}\text { Fuente de } \\
\text { aprendizaje }\end{array}$ \\
\hline COMPETENCIAS-HABILIDADES & $\begin{array}{c}\text { Baja Alta } \\
123456789\end{array}$ & $\begin{array}{c}\text { Baja Alta } \\
123456789\end{array}$ & $\begin{array}{c}\text { Cl Clase } \\
\text { Co Cursos } \\
\text { B Bibliotecas } \\
\text { A Autodicacta } \\
\text { 0 Otros }\end{array}$ \\
\hline BÚSQUEDA DE INFORMACIÓN & & & \\
\hline 1. Fuentes de información impresas (libros, artículos,...) & & & \\
\hline $\begin{array}{l}\text { 2. Acceso y uso de catálogos automatizados } \\
\text { 3. Consulta y uso de fuentes electrónicas de información } \\
\text { primaria (libros, ...) }\end{array}$ & & & \\
\hline $\begin{array}{l}\text { 4. Consulta y uso de fuentes electrónicas de información } \\
\text { secundaria (bases de datos) }\end{array}$ & & & \\
\hline 5. Conocimiento de la terminología de tu materia & & & \\
\hline $\begin{array}{l}\text { 6. Búsqueda y obtención de información en internet } \\
\text { (búsqueda avanzada, directorios, portales) }\end{array}$ & & & \\
\hline $\begin{array}{l}\text { 7. Uso de fuentes de información electrónicas informales } \\
\text { (blogs, listas de discusión...) }\end{array}$ & & & \\
\hline $\begin{array}{l}\text { 8. Conocimiento de las estrategias de búsqueda de } \\
\text { información (descriptores, operadores booleanos,...) }\end{array}$ & & & \\
\hline
\end{tabular}




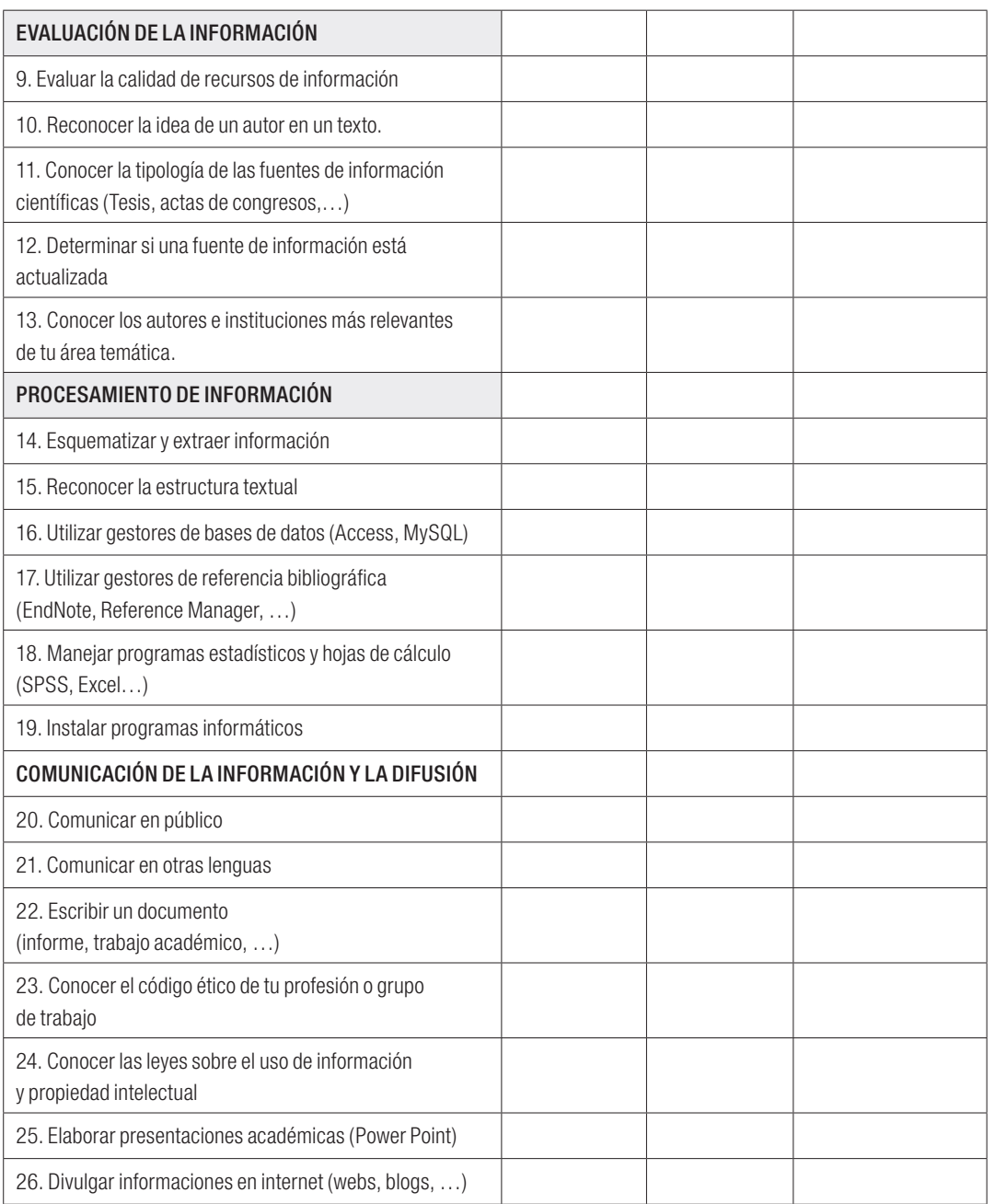

Señala algunas necesidades que consideras relevantes para tu formación académica y para alcanzar una mejor competencia informacional: 